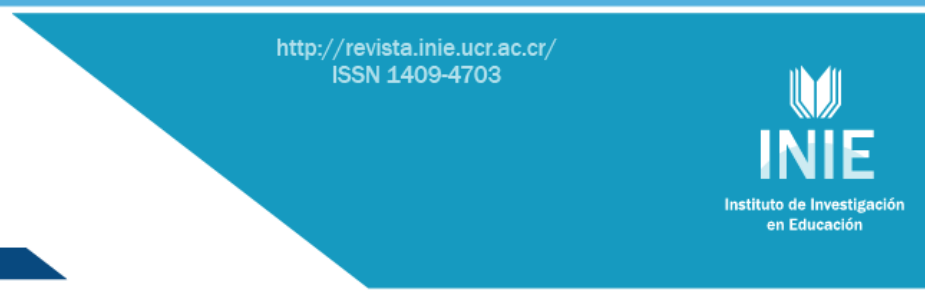

\title{
FACTORES QUE INCIDEN EN LAS CONDICIONES DE EMPLEABILIDAD DE LOS EGRESADOS DE PEDAGOGÍA
}

FACTORS AFFECTING CONDITIONS OF EMPLOYABILITY AMONG GRADUATES OF TEACHER PROGRAMS

\author{
Volumen 16, Número 1 \\ Enero - Abril \\ pp. 1-19
}

Este número se publicó el $1^{\circ}$ de enero de 2016

DOI: http://dx.doi.org/10.15517/aie.v16i1.21935

\author{
Orietta Geeregat Vera \\ Gonzalo Cifuentes Gómez \\ Mari Carmen Villarroel Farías
}

Revista indizada en $\underline{\text { REDALYC, SCIELO }}$

Revista distribuida en las bases de datos:

LATINDEX, DOAJ, E-REVIST@S, IRESIE, CLASE, DIALNET, SHERPA/ROMEO, QUALIS, MIAR

Revista registrada en los directorios:

ULRICH'S, REDIE, RINACE, OEI, MAESTROTECA, PREAL, CLACSO 


\title{
FACTORES QUE INCIDEN EN LAS CONDICIONES DE EMPLEABILIDAD DE LOS EGRESADOS DE PEDAGOGÍA FACTORS AFFECTING CONDITIONS OF EMPLOYABILITY AMONG GRADUATES OF TEACHER PROGRAMS
}

\author{
Orietta Geeregat Vera ${ }^{1}$ \\ Gonzalo Cifuentes Gómez ${ }^{2}$ \\ Mari Carmen Villarroel Farías ${ }^{3}$
}

\begin{abstract}
Resumen: En Chile, tras 30 años de liberalización del sistema de Educación Superior, la oferta de carreras de pedagogía se ha incrementado sobre el resto de las carreras, lo que ha constituido uno de los factores en la disminución de los niveles de exigencia al ingreso. Bajo este contexto y ante las críticas a la calidad de los programas de formación de profesores y las competencias que estos desarrollan durante la carrera, surge el cuestionamiento de identificar, además de la calidad de los programas de formación, qué otros elementos influyen en las condiciones de empleabilidad de los titulados de pedagogía. En este sentido, el ensayo operacionaliza el constructo empleabilidad, revisa sus distintas formas de medición y elabora una propuesta al respecto. Entre los principales resultados es posible precisar que el constructo empleabilidad reúne una serie de factores dinámicos que han sido clasificados en externos al sujeto e internos a él. Algunos externos son el prestigio de la universidad, la influencia del establecimiento educacional de origen y el salario. Entre los factores internos, se describe la influencia de los resultados en pruebas estandarizadas, la adaptación al contexto y la percepción de autoeficacia, entre otras. Los factores recién descritos, han sido regularmente observados de forma independiente, lo que a juicio de los autores, limitaría las opciones de análisis del fenómeno. Por lo anterior, se propone una mirada que integre dichos factores y considere elementos de contexto que permitan estudiar la empleabilidad como un constructo complejo, considerando la influencia que ejerce el entorno.
\end{abstract}

Palabras clave: FORMACIÓN DE PROFESORES, FACTORES DE EMPLEABILIDAD, PEDAGOGÍA, CHILE.

Abstract: In Chile, after 30 years of liberalization of the system of higher education, teaching program offer has increased over other programs, which has been one of the factors in decreasing the requirements for enrollment. In this context and given the criticism of the quality of teacher training programs and skills they develop during the forming years, the question arises to identify, besides the quality of the training programs, what other elements influence the teaching program graduates' employability conditions. In this sense, this essay operationalizes the construct employability, reviews different forms of measurement and develops a measure proposal. Among the main results it can be noted that the employability construct has a number of dynamic factors that have been classified into external and internal to the subject. The external ones describe emphasize prestige of the university, influence of the educational institution of origin and salary. The internal factors describe the influence of standardized tests results, adaptation to the context and perception of self-efficacy, among others. Factors described above, have been regularly observed independently, which in the opinion of the authors, would limit the options to analyze the phenomenon. In this sense, the proposal is to have a view which integrates these factors and consider contextual elements that permit studying employability as a complex construct, considering the influence of the environment.

Key words: TEACHERS' TRAINING, EMPLOYABILITY FACTORS, PEDAGOGY, CHILE.

\footnotetext{
1 Académica Asociada Jornada Completa de la Universidad de La Frontera

(UFRO), Temuco-Chile. Dirección electrónica: orietta.geeregat@ufrontera.cl

2 Diseñador Instruccional en el Centro de Educación a Distancia-TELEDUC, Universidad Católica de Chile. Dirección electrónica: gecifuentes@uc.cl

3 Académica de la Universidad de La Frontera, Temuco-Chile. Dirección electrónica: mari.villarroel@ufrontera.cl
}

Ensayo recibido: 2 de marzo, 2015

Enviado a corrección: 16 de junio, 2015

Aprobado: 9 de noviembre, 2015 


\section{Introducción}

La sociedad demanda de las nuevas generaciones de profesores requerimientos más amplios y complejos que hace unas décadas atrás. Dichos requerimientos han transitado desde la enseñanza de un conocimiento factual al dominio de estrategias didácticas que les brinden a las nuevas generaciones las oportunidades de aprendizajes necesarias para desenvolverse con éxito en la sociedad del conocimiento, ello implica que los profesores consideren en sus prácticas la diversidad socio-cultural, la integración de las nuevas tecnologías, la evaluación como parte del aprendizaje, el trabajo en equipo, entre otras (Cox, 2006).

A nivel internacional los países con mejores resultados educacionales se enfocan en lograr una buena atracción, selección y formación inicial de los profesores pero al mismo tiempo se preocupan tanto de la formación continua de su cuerpo profesional como de la forma en que estos se involucran en el proceso de formación e inducción de las nuevas generaciones de profesores (Barber y Mourshed, 2008). Responder a estas exigencias demanda a las instituciones responsables de la Formación Inicial Docente (FID) la capacidad de implementar una serie de acciones que consolide la triada: instituciones formadoras, Ministerio de Educación (MINEDUC) y sistema escolar (Cox, 2006).

En Chile, transcurridos 30 años de la creación de un pseudo mercado en la educación superior, es posible apreciar una expansión desregulada de los programas de formación de profesores y por ende del número de sus titulados. Por ejemplo, entre los años 2000 y 2008 la matrícula de las carreras de pedagogía creció en un 157\%, mientras que en el resto de las carreras aumentó en un $94 \%$, lo que se asocia a una disminución de los niveles de exigencia para el ingreso (Meckes y Bascopé, 2010).

Bajo este contexto, tal parece que las asignaturas ofrecidas por las instituciones responsables de la FID no va al mismo ritmo de cambio del parámetro internacional, lo que se traduce principalmente en una separación entre el proceso de formación de profesores y el mundo escolar (Latorre, 2005), repercutiendo así en las condiciones de empleabilidad de los recién egresados.

En consideración de lo anterior, el presente ensayo ${ }^{3}$ pretende identificar qué otros elementos, además de la calidad de los programas de formación de profesores, influyen en

\footnotetext{
${ }^{3}$ Este trabajo es productividad de los autores del Proyecto DI14-0063 "Estudio analítico-descriptivo sobre las condiciones de empleabilidad de los titulados de pedagogía de la Universidad de La Frontera..."(2014-2015)
} 
las condiciones de empleabilidad de los titulados de pedagogía, a partir de la operacionalización de este constructo y sus factores asociados.

\section{Nudos críticos y opciones para la acción}

El contexto del siglo XXI, asociado a las características de la sociedad del conocimiento como eje articulador, implica un cambio paradigmático en cuanto a la profesión docente en donde se transita desde un profesional "que detenta el conocimiento" y se lo entrega a alguien menos avezado en la cultura, hacia un profesional capaz de "crear y orquestar ambientes de aprendizaje complejos", lo que implica involucrar a los estudiantes en actividades apropiadas, de manera que estos puedan construir su propia comprensión del contenido. A pesar de este cambio paradigmático, existe una separación entre el proceso de formación de profesores y el mundo escolar (Latorre, 2005). Al respecto, Álvarez (2012) señala que la universidad y la escuela generan distintos tipos de conocimiento con diferentes niveles de especialización, lo cual se manifiesta en una formación inicial altamente teórica, en contraste con los centros escolares en donde suelen generarse culturas profesionales con menor apoyo teórico. A lo anterior, se suma la diferencia en términos de conocimientos prácticos que existe entre los docentes de aula y los docentes universitarios.

Las tendencias de los sistemas de formación docente en la primera década del siglo XXI se han orientado principalmente en dos direcciones: la primera, relacionada con aumentar los niveles de exigencia, asociados a los conocimientos y competencias adecuadas para la enseñanza escolar, con la intención de reducir la brecha entre lo que hacen las instituciones formadoras y los requerimientos de la práctica de la enseñanza en este. Un segundo camino se asocia al alza de estándares que tienen como nombre genérico la profesionalización. Esta última es criticada tanto desde las unidades educativas como desde los ministerios de educación debido a su carácter teórico y alejado de las necesidades particulares de la enseñanza (Cox, Beca y Cerri, 2011; Darling-Hammond y Bransford, 2005).

Respecto a la separación entre la teoría y la práctica en la FID, es interesante analizar cómo los profesores recién titulados se insertan en el mundo laboral y plasman en su ámbito de acción profesional la formación recibida. En este sentido, se propone discutir en torno al constructo empleabilidad y los factores que influyen en él y cómo estos influyen en su forma de medición. 


\section{Constructo Empleabilidad}

Enríquez y Rentería (2007) concluyen que empleabilidad es un constructo que reúne una serie de características dinámicas que evolucionan en relación al contexto en que se encuentran inmersos los individuos. Así por ejemplo, lo que era deseable para un trabajador a inicios del siglo XX es distinto a lo requerido en el contexto del siglo XXI. Adicionalmente, influyen el lugar en donde se requiera el empleo y el sector de la economía en donde de desempeñará el individuo.

En esta misma línea de aproximación, existe una corriente que estudia el constructo empleabilidad como un conjunto de factores intrínsecos al individuo que aumentan o disminuyen las opciones de conseguir un empleo. Aquí encontramos autores como Castañeda y Sánchez (2009), quienes asocian dicho constructo a la gestión que el propio individuo realiza de su desarrollo profesional, en donde consideran las condiciones del mercado, la proactividad ante las oportunidades y la ampliación permanente de las áreas de competencia del individuo a través de una formación continua.

Vargas (2008) relaciona el constructo empleabilidad con las actitudes individuales para obtener y conservar un empleo más allá de las condiciones que el puesto de trabajo requiere en sí mismo. En este sentido, las condiciones de empleabilidad se asocian más al contexto sociocultural del individuo y, por ende, sus condiciones de entrada, más que el nivel de escolaridad alcanzado.

Para la Organización Internacional del Trabajo (OIT), el constructo empleabilidad se entiende como el conjunto de conocimientos y competencias que aumentan la capacidad de los trabajadores para conseguir y conservar un empleo. Dichos conocimientos y competencias se expresan en una mejora permanente de su trabajo y en la adaptación al cambio que manifiestan (Molpeceres, 2004).

Aliaga y Schalk (2010) asocian el constructo empleabilidad a un conjunto de capacidades básicas que contribuyen a un desempeño eficiente en un puesto de trabajo. Dichas capacidades las clasifican en: capacidades de comunicación, de relación interpersonal, de resolución de problemas, de manejo de procesos organizacionales y de regulación de los propios comportamientos con base en los requerimientos de la actividad profesional.

Para Thieme (2007) el constructo empleabilidad se relaciona a competencias genéricas, las que deben ser transversales, transferibles y por ende, con procesos de adquisición observables. 
En Chile, diversos estudios (Rojas, Chávez, y Suazo, 2011; Sánchez, Fuller, y López, 2010) se han centrado en analizar el impacto que ha tenido en la empleabilidad y la inserción al mundo laboral, el desarrollo de competencias genéricas durante el proceso de formación. De estos estudios se concluye que una aproximación efectiva al constructo está dado por la medición de competencias como: "trabajo en equipo", "comunicación oral y escrita en español”, “empatía”, “creatividad” y "responsabilidad".

Desde otra perspectiva, existe una aproximación al constructo en donde se considera la influencia de factores externos, como las condiciones generales del mercado laboral (CEOC, 2010; Enríquez y Rentería, 2007), el prestigio de la universidad, el salario ofrecido, entre otras (ver punto 5.1).

De las definiciones recopiladas es posible observar una diferencia entre los autores que ponen el foco en factores contextuales de aquellos que centran la mirada en aspectos intrínsecos al individuo. Estos factores se relacionarían con las condiciones de entrada al proceso de formación profesional o bien a la manera que dichos centros de formación desarrollan en los estudiantes las competencias necesarias para afrontar con éxito las condiciones impuestas por el mercado laboral.

Desde esta perspectiva cabe preguntarse cómo se puede medir y analizar la empleabilidad en un contexto laboral como el docente en donde existe una particular regulación.

En Chile, el mercado laboral en educación se estructura en torno a dos grandes grupos. Por una parte están aquellos establecimientos educacionales que dependen de forma directa tanto en términos económicos como administrativos del Estado y por otra, existe un grupo de establecimientos que si bien depende en términos económicos del Estado (a través de la subvención a la matricula), en términos administrativos depende de sostenedores privados.

Bajo este régimen y más allá de los esfuerzos del Estado de Chile por regular el mercado de la educación, es posible distinguir con claridad un grupo de profesores que en tanto dependientes económica y administrativamente del Estado, son regulados por un "Estatuto Docente", el cual les asegura mejores condiciones laborales que aquellos docentes que trabajan en establecimientos subvencionados, quienes dependen del "Código del Trabajo" al igual que el resto de los trabajadores del país. Así por ejemplo, bajo este último régimen existe la posibilidad de despedir a un profesor por "necesidades de la empresa". 
Esta dispar situación, en términos contractuales, podría hacer pensar que las competencias buscadas por uno u otro empleador (Estado o Particulares) pueden ser distintas, independiente de la existencia de un marco nacional (Marco para la Buena Enseñanza) que explicita lo que es deseable de un buen profesor.

En consideración de lo anterior, se propone una medición y análisis de la empleabilidad en el contexto educativo chileno que integre indicadores objetivos, asociados al mercado laboral e indicadores particulares de los individuos. Lo anterior permitirá observar las interacciones entre la formación recibida en la universidad con lo esperado por los empleadores en contextos situados concretos.

\section{Competencias valoradas por los empleadores.}

El tránsito desde el egreso de la universidad al mundo laboral suele pensarse como un proceso lineal, sin embargo, el mercado laboral tiene características diversas en donde intervienen una serie de factores de carácter objetivos, subjetivos, personales y contextuales que resultan difíciles de anticipar y controlar (Rojas et al., 2011). A estos factores se suma la poca preparación que tienen los profesionales en tres áreas claves: expresión oral y escrita, habilidades interpersonales y adaptación al mundo laboral. El estudio del Centro de Medición MIDE-UC (2008) concluye que la adquisición de estas herramientas por parte de los profesionales noveles, es uno de los principales desafíos del sistema educativo chileno.

Desde la perspectiva del empleador, el estudio de Martínez (2009) señala que estos en términos generales valoran las cualidades personales del futuro trabajador por sobre las habilidades laborales y conocimientos técnicos. A su juicio, serán estas cualidades personales, las que convierten a alguien en "confiable" y con la capacidad de adaptarse a la cultura de la empresa.

En esta misma línea, el estudio realizado por el MIDE-UC (2008) concluye que los empleadores cuando contratan a un recién egresado, en primer lugar centran su atención en competencias de corte ético, como el compromiso con la calidad de la labor profesional realizada y la capacidad para aplicar los conocimientos adquiridos en la práctica. En segundo lugar valoran la capacidad para reconocer, plantear y resolver problemas y finalmente destacan habilidades sociales tales como el trabajo en equipo y las habilidades interpersonales. Por el contrario, los factores menos valorados son el dominio de un idioma extranjero y las notas que obtuvo el postulante durante la carrera, ya que en este último punto prima el "prestigio social" de la institución (ver punto 5.1.1). 
Para el caso de los profesores, se demandan principalmente "habilidades relacionales", que les permitan enfrentar con éxito los desafíos que impone el trabajo en el aula. En este sentido, los empleadores en general opinan que los profesionales recién egresados tienen una buena preparación académica, sin embargo, para el caso de los profesores, estos consideran que poseen un deficiente conocimiento conceptual, tanto de la disciplina que enseñan, como en la didáctica requerida para ello (MIDE-UC, 2008).

\section{Factores que Influyen en la empleabilidad de los profesores nóveles}

Para el profesor, el tránsito hacia el mundo laboral se torna más dificultoso si se considera el bajo impacto que tiene la FID en los recién titulados (Ávalos, 2004; Manzi, 2010; MINEDUC, 2005; OCDE, 2004). Dicho impacto se expresa en las primeras aproximaciones en el aula, en donde el profesor novel tiende a refugiarse en su experiencia como estudiante, a partir de la cual imita las prácticas pedagógicas de sus profesores o bien, se somete a las demandas de su contexto laboral (Latorre, 2005), para lo cual deja de lado los conocimientos adquiridos en su formación universitaria.

Resulta evidente que un recién titulado posee un nivel de competencias que se asocia a las de un novato en su profesión. Al respecto Corvalán (2010) señala que para alcanzar un mayor nivel pueden llegar a transcurrir entre cinco y siete años de práctica profesional activa. En este periodo, Cornejo (1999) reconoce dos fases; la primera, denominada "umbral o antesala" que se extiende entre el primer y el sexto mes de docencia; la segunda, denominada "madurez y crecimiento" se extiende entre los tres y cinco años de ejercicio de la profesión.

Para Vonk (1996) la etapa inicial de la profesión docente constituye un proceso de inducción que se compone de dos momentos claves: "ambientación” y "progresión”, en donde el recién titulado debe asumir que está en una transición desde un "sujeto en formación" hasta la de un "profesional autodirigido". Finalmente, Imbernón (1998) entiende esta etapa como un proceso de "socialización" en la que el profesor recién titulado debe integrarse como miembro activo y participante del colectivo profesional.

A partir de la revisión de la literatura nacional, es posible identificar una serie de factores más o menos comunes que inciden en los profesores nóveles al momento de encontrar su primer trabajo y mantenerse en él. A continuación, se presenta una 
organización de los factores más relevantes respecto de la empleabilidad de un egresado de pedagogía 4 .

\subsection{Factores externos al profesor novel}

\subsubsection{Prestigio de la Universidad}

Tanto los estudios de Ruffinelli y Guerrero (2009) como los del MIDE-UC (2008), señalan que en la percepción de los empleadores, las universidades creadas previo a la década de los 80 cuentan con un mayor prestigio que las creadas posteriormente; para los autores, llama la atención que dicha percepción no depende de las características de las nuevas universidades, en términos de los grados académicos de sus docentes o la preparación de sus titulado, sino en la idea instalada de que ciertas universidades poseen "mejor calidad" dependiendo de si estas fueron creadas antes o después de las reformas de la década del 80.

Dichas diferencias de percepción se manifiestan al momento de postular a un empleo debido a la asociación que realizan los empleadores entre el "prestigio social" de la universidad y la calidad de los egresados de estas universidades, independiente de las calificaciones o competencias específicas de estos profesionales. Esta asociación finalmente influye en las opciones laborales de los titulados de pedagogía.

\subsubsection{Selectividad de la institución formadora}

Asociado al punto anterior, el estudio de Meckes y Bascopé (2010) logró determinar una correlación positiva entre el nivel de selectividad de la institución de egreso y la probabilidad de sus titulados para trabajar en un establecimiento del sector subvencionado. No obstante, es importante considerar que ingresar a una universidad selectiva no se relaciona necesariamente con la obtención de altas calificaciones. De esta manera, el prestigio de la institución ejerce una gran influencia sobre los empleadores sin importar las calificaciones de los postulantes.

\footnotetext{
${ }^{4}$ Es importante señalar que los estudios a los que se hará referencia analizan a titulados de Pedagogía en Educación Básica, por lo que para efectos de una primera aproximación y ante la falta de estudios en este ámbito para titulados de Pedagogía en Enseñanza Media serán considerados dichos factores como referentes en el desarrollo de un marco referencial.
} 


\subsubsection{Influencia del establecimiento educacional de origen}

Diversos autores (Meckes y Bascopé, 2010; Ruffinelli y Guerrero, 2009) dan luces respecto de una segmentación en el mercado laboral docente, en donde jóvenes egresados de colegios privados tienden a estudiar en universidades de mayor prestigio social, para finalmente ejercer su profesión en colegios privados. Por el contrario, los jóvenes que estudiaron en establecimientos de dependencia municipal tienden a estudiar en universidades de menor prestigio, y finalmente se insertan laboralmente en establecimientos de carácter municipal o subvencionado de bajo nivel socioeconómico.

\subsubsection{Salario}

Tanto el salario como las condiciones laborales influyen en la decisión de un profesor novel para aceptar un determinado trabajo. A su vez, los empleadores seleccionan a los postulantes a partir del equilibrio que existe entre el salario que pueden ofrecer y sus preferencias respecto de las características de determinados profesores (Meckes y Bascopé, 2010).

\subsection{Factores internos al profesor novel}

\subsubsection{Resultados en Pruebas Estandarizadas}

Un factor importante es el puntaje que obtienen los estudiantes en la Evaluación Inicia ${ }^{5}$. Con base en una muestra del $60 \%$ al $80 \%$ de los egresados de Pedagogía Básica del año 2008, Meckes y Bascopé (2010) concluyen que la probabilidad de ser contratado por colegios de menor nivel socioeconómico o de dependencia municipal disminuye en la medida que aumenta el porcentaje de logro que se obtuvo en la INICIA de conocimientos disciplinarios.

En consecuencia, los profesores noveles que obtienen un mejor rendimiento en la Evaluación Inicia tienden a ubicarse en escuelas de mayor nivel socioeconómico y con mejores resultados en el Sistema Nacional de Evaluación de Resultados de Aprendizaje (SIMCE). Por el contrario, aquellos profesores que son primera generación de universitarios en sus familias tienden a ubicarse en escuelas con menores puntajes SIMCE. Es preciso

\footnotetext{
${ }^{5}$ La Evaluación Inicia es un sistema creado por el Ministerio de Educación de Chile, cuyo propósito es verificar la calidad de los programas de FID. Dicho sistema es integrado por una batería de pruebas que rinden de forma voluntaria los egresados de las carreras de Educación Parvularia, Pedagogía en Educación Básica y Pedagogía en Educación Media.
} 
señalar que el estudio no indica que el puntaje sea un dato que analicen los directivos para contratar, sino que se encontró una correlación entre ambos factores.

En esta misma línea, el estudio de Ávalos (2009) propone una clasificación respecto a macro y micro factores que influyen en la empleabilidad de los titulados de pedagogía, en donde los macro factores se asocian con la inserción de lógicas de mercado en la obtención y mantención en un puesto de trabajo. En este sentido, la valoración de un profesor pasa por los resultados que logran sus estudiantes en pruebas estandarizadas (SIMCE, PSU). Así, un profesor cotizado en el mercado laboral docente será aquel que pueda acreditar no solo buenos resultados con sus estudiantes, sino que también tenga buenos resultados en la Evaluación Docente del Ministerio de Educación (MINEDUC).

\subsubsection{Capital cultural}

En términos sociológicos, Ávalos, Carlson y Aylwin (2004) hacen referencia a los planteamientos de Bourdieu y Passeron (1973), respecto de la influencia del capital cultural transmitido por el hogar a los profesores noveles. De este modo, quienes poseen mayor capital cultural también acumulan mayor capital escolar a lo largo de su trayectoria académica. De manera que son estos jóvenes los que acceden a universidades de mayor reputación, por lo que capitalizan, al momento de ingresar al mercado laboral, todo el conocimiento acumulado a lo largo de la vida, lo que se expresa en una mayor probabilidad de acceder a cargos de mayor reputación.

\subsubsection{Redes de contacto}

Asociado al punto anterior, existe una relación entre el capital social y las redes de contacto que logran generar los profesores noveles durante su periodo universitario. Eso sí, se debe considerar que la posibilidad de generar estas redes se encuentran estrechamente relacionadas al nivel de selectividad de la institución en la que cursaron sus estudios (Mayer y Youngs, 2009 citado en Meckes y Bascopé, 2010).

\subsubsection{Calificaciones obtenidas en la FID}

A diferencia de lo planteado por los estudios de Ruffinelli y Guerrero (2009) y del MIDE-UC (2008) (ver punto 5.1.1), el estudio de Cabezas, Gallego, Santelices y Zarhi (2011) hace referencia a la evidencia que existe en Chile respecto a la influencia de las calificaciones en el trabajo que se obtiene. En términos concretos, los autores demostraron 
que existe una desigual distribución de las calificaciones de los estudiantes de pedagogía en relación con el trabajo que obtienen. Es así como en aquellos establecimientos con menores niveles socioeconómicos tienden a concentrarse los profesores con menores calificaciones.

\subsubsection{Adaptación al contexto}

La llegada de un nuevo profesor a una escuela significa una experiencia de inserción distinta, producto de las diferencias que existen en términos de infraestructura y del tipo de estudiante al que atiende, por ejemplo, entre una escuela de dependencia municipal, comparada con una escuela subvencionada con selección y alto copago. Así, la forma en que los profesores noveles sortean las dificultades que el medio les impone constituye un factor importante respecto a mantenerse en un determinado establecimiento y lograr prestigio al interior de él (Ávalos, 2009).

A modo de ejemplo, Ávalos (2009) cita el estudio de Johnson y Kardos (2004) realizado en Estados Unidos, en este compararon la inserción de nuevos profesores en escuelas con población socio-económica vulnerable versus las escuelas con estudiantes de nivel más alto. Del estudio se concluye que en los establecimientos de mayor vulnerabilidad se esperaba que los profesores y las profesoras recién egresados actuaran como expertos, por lo que no se les consultaba si requerían de apoyo y eran aislados en sus aulas, lo que hacía su proceso de adaptación más dificultoso.

A nivel nacional, los profesores noveles reconocieron que cuando iniciaron su trabajo, la mayor parte del tiempo no contaron con un apoyo formal, a excepción de contadas oportunidades en que profesores de mayor experiencia se mostraron dispuestos a ayudar (Ávalos et al., 2004).

\subsubsection{La autoeficacia}

La autoeficacia es definida por Bandura (1977; citado en Tagle, Del Valle, Flores y Ackley, 2012, p. 2) como "las creencias sobre las propias capacidades para organizar y desarrollar cursos de acción requeridos para producir logros determinados". En este sentido, la autoeficacia se refiere a la confianza de una persona en torno a la capacidad para hacer las actividades que emprende (Velásquez, Martínez y Cumsille; 2004).

Para Bandura (1997, citado en Velásquez, Martínez y Cumsille, 2004) los pensamientos de autoeficacia se derivan de cuatro fuentes: (a) las experiencias de éxito (b) los efectos producidos al observar acciones de los demás (c) la exposición a los juicios 
verbales de los demás que actúan como persuasión social y (d) los estados emocionales y fisiológicos (estrés, excitación, fatiga).

Para el caso de los docentes, la autoeficacia se define como las prácticas de los maestros respecto a las habilidades que poseen para producir un efecto positivo en el aprendizaje de sus estudiantes (Drinot, 2012). En esta línea, el estudio de Tagle et al. (2012) hace una extensa revisión bibliográfica que da cuenta de cómo los docentes responden a situaciones complejas en la sala de clases a partir de sus creencias personales 0 autoeficacia. En este contexto existen diversas investigaciones que han establecido una relación entre la autoeficacia de los docentes con los niveles de logro de los estudiantes.

Montecinos, Barrios y Tapia (2011) señalan por su parte que, una de las influencias más potentes en la percepción de autoeficacia en los profesores, son las experiencias exitosas que han tenido, tanto en la práctica profesional como durante el primer año de inserción laboral,de ahí que es interesante explorar la influencia que este factor interno podría tener en las condiciones de empleabilidad de los profesores noveles.

\section{Propuesta para la observación del constructo empleabilidad}

La literatura coincide en señalar que la empleabilidad se asocia a factores tanto internos como externos al individuo. No obstante, estos factores tienden a ser vistos de manera independiente, lo que dificulta la observación de relaciones que podrían brindar una compresión más contextualizada del fenómeno. Analizar el constructo como un conjunto de factores internos y externos interrelacionados permite abrir la forma de aproximarnos al fenómeno de estudio y establecer relaciones teóricas entre unos y otros, los que posteriormente podrán ser comprobados o descartados.

En este contexto, es importante considerar la fuerte influencia que ejerce el capital cultural del individuo (factor interno) en una serie de otras características que se relacionan con la empleabilidad. Dicho capital cultural, al momento de ingresar a la universidad, no solo ya está consolidado, sino que además influye en otros factores de carácter externo, tales como el establecimiento educacional de origen y el ingreso a universidades de prestigio (Ver esquema 1). 
Esquema 1.

Relación de los factores que influyen en la empleabilidad de los docentes

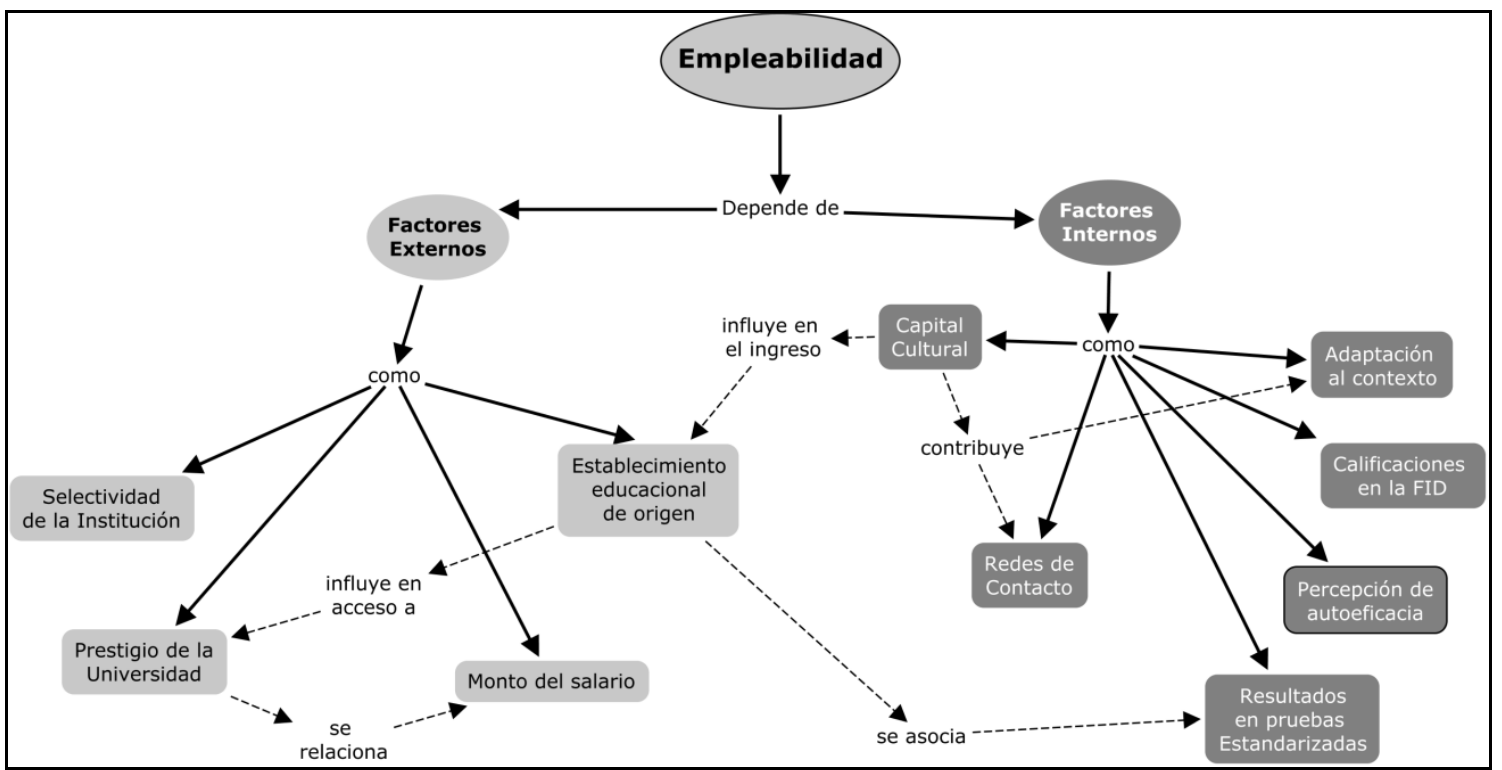

Fuente: Elaboración propia.

Al respecto, sería interesante revisar las condiciones de entrada de los estudiantes de pedagogía, en términos de capital cultural, y ver si éstas se relacionan a las condiciones de empleabilidad posteriores. Así por ejemplo, se podría profundizar en este punto o bien en analizar qué tan determinantes fueron dichas condiciones, sobre la formación recibida en la universidad, para lograr o no la obtención de un empleo. En este sentido, es importante observar la influencia que ejerce en la empleabilidad, las redes de contacto, capital cultural, mejores calificaciones o mejores resultados en pruebas estandarizadas, tal como lo indica la literatura. O bien, si estos factores no son relacionables y existen otros que no han sido observados. Al respecto se presume que podrían estar influyendo una mezcla de factores, por ende, más que una relación lineal entre uno y otro, la obtención de un empleo dependería de la sumatoria de estos factores; los que unidos crean las condiciones necesarias para la obtención de un empleo y el desarrollo de una carrera al interior de la institución educativa.

Otro elemento interesante a considerar es la influencia que ejerce la percepción de autoeficacia respecto a las condiciones de empleabilidad, por ejemplo observar la existencia o no de una asociación entre aquellos profesores que poseen una percepción de 
autoeficacia más alta, respecto a los factores internos y/o externos descritos y cómo esto finalmente se relaciona a las condiciones de empleabilidad.

En esta misma línea, es importante considerar algunos resguardos si se desea explorar una relación entre empleabilidad y autoeficacia. Por ejemplo, el estudio de RimmKaufman y Sawyer (2004) hace referencia a la influencia que ejerce el medio en la percepción de autoeficacia, de ahí que si un docente trabaja en un contexto escolar con creencias y prácticas pedagógicas altamente arraigadas es altamente probable que finalmente primen estas por sobre sus creencias, afectando su percepción de autoeficacia. Complementariamente, el estudio de Drinot (2012) hace referencia a los límites del propio constructo de autoeficacia, poniendo una cortapisa a las conclusiones que son posibles de extraer a partir de él, toda vez que por más que un individuo posea una alta percepción de autoeficacia y en consecuencia, se crea capaz de realizar una determinada tarea, dicha percepción no puede ir más allá de sus capacidades reales, pues como es lógico, requiere de las habilidades y conocimientos necesarios para lograr desarrollar la tarea con éxito.

Desde el punto de vista del diseño de investigación, es importante que éste permita aproximar al investigador a entender cómo estudiantes con menor capital cultural, que cursan la FID en universidades de menor prestigio social (comparado con las universidades tradicionales del área Metropolitana), logran no sólo conseguir un empleo al poco tiempo de titulado, sino que además, son exitosos en sus instituciones, lo que se plasma en un reconocido prestigio entre sus pares.

Este tipo de situaciones refuerza la idea de que el conjunto de estos factores (externos e internos), dependientes de las condiciones de entrada y de contexto del individuo, influye en la formación de un profesional con las competencias necesarias para obtener y mantenerse en un empleo.

El constructo empleabilidad docente en la capital del país (Santiago), con sus particulares condiciones del mercado laboral, puede ser diametralmente distinto al de una región con altos niveles de ruralidad y pobreza, en donde dichas condiciones podrían influenciar el resto de los factores que la literatura identifica como intervinientes. En este sentido, un estudiante que egresa de un establecimiento de enseñanza media, de dependencia particular subvencionada y que ingresa a una universidad de menor prestigio en comparación con los planteles tradicionales de la capital del país, debería tener menores opciones de obtener un empleo, no obstante ello no ocurre. En este sentido, podríamos plantearnos las siguientes preguntas: ¿Qué factores influyen entonces? ¿Los empleadores 
en este contexto privilegian otras características de los profesores recién titulados? ¿La universidad influye de manera decisiva en las competencias laborales de los estudiantes a diferencia de lo que sucede en las zonas del país más favorecidas económicamente? ¿Existe un mercado laboral docente segregador, en donde cierto tipo de estudiantes obtiene trabajos en un tipo de establecimientos de mejores condiciones, en desmedro del otro grupo de estudiantes?

\section{Síntesis y Reflexiones Finales}

La literatura da cuenta de distintos factores que influyen en las condiciones de empleabilidad de los docentes, dichos factores de carácter interno y externo van desde miradas economicistas del individuo (proactividad, capacitación permanente) hasta otras de corte social (prestigio de la universidad, calificaciones). Dichos factores han sido observados de manera independiente, lo que a juicio de los autores limita las opciones de análisis del fenómeno. En este sentido, se propone una mirada que integre estos factores y considere elementos de contexto que permitan estudiar la empleabilidad como un constructo complejo, considerando la influencia que ejerce el contexto de estudio.

Es legítimo preguntarse respecto a la forma cómo las instituciones formadoras de profesores pueden hacerse cargo de las condiciones de empleabilidad de sus titulados y brindar las oportunidades de aprendizaje para que estos mejoren sus competencias, independientemente del contexto sociocultural de procedencia.

El desafío que impone hoy la profesión docente exige de los profesores la construcción de aprendizajes centrados en sus estudiantes, sin embargo, estos últimos son obligados a ser parte de un sistema educacional que guarda poca relación con el contexto sociocultural en el que habitan. En este sentido, numerosas investigaciones (Bustos y Coll, 2010; Cox, 2006; Cox, et al., 2011; Haydn y Barton, 2005; Silva, Gros, Garrido y Rodríguez, 2006; Twining, Broadie, Cook, Ford, Morris, Twiner y Underwood, 2006; Walsh, 2002) tanto a nivel nacional como internacional señalan que los docentes deben responder a las necesidades de aprendizaje individual de los estudiantes. Así, los programas de FID deben formar a un profesional que sea experto en su disciplina, que domine los estándares mínimos que propone el Ministerio de Educación y que además posea una serie de competencias, las cuales aparentemente aumentarán sus posibilidades de encontrar trabajo.

No obstante, en la actualidad, el foco de la FID está en el fortalecimiento de los aspectos pedagógicos (didáctica general y del contenido) y no en el desarrollo explícito de 
competencias genéricas que apunten a mejorar la empleabilidad de sus egresados. En este sentido, aparentemente, los profesores noveles que, en promedio logran una temprana y exitosa empleabilidad, se debe a las características particulares de su entorno sociocultural, más que a las herramientas dadas por la universidad durante su formación.

Entonces ¿Cómo traspasar, efectivamente, dichas "habilidades particulares" que influyen en la empleabilidad a estudiantes de pedagogía que no han logrado desarrollarlas en sus hogares o en 12 años de escolaridad? ¿Cómo brindarles oportunidades de aprendizaje reales a estos estudiantes sin descuidar el proceso de formación docente?

Desde hace décadas en la FID se ha buscado la fusión de la disciplina con la pedagogía, sin embargo tanto a nivel internacional como nacional, la tendencia es la separación de estos procesos, con departamentos y académicos especialistas para cada área sin considerar las nuevas corrientes en FID que hablan de un diálogo permanente entre los distintos conocimientos que requiere la profesión docente (Shulman, 1987). En este contexto, cabe preguntarse ¿Cómo incluir el desarrollo de estas competencias de empleabilidad que parecieran estar determinadas por el contexto y las particularidades de los individuos a un proceso de formación docente estandarizado?

\section{Referencias}

Aliaga, Claudia y Schalk, Ana. (2010). E2: empleabilidad temprana y emprendimiento. Dos grandes desafíos en la formación superior en chile. Calidad en la Educación (33), 319337.

Álvarez, Carmen. (2012) ¿Qué sabemos de la relación entre la teoría y la práctica en la educación? Revista Iberoamericana de Educación, (60/2), 1-11.

Ávalos, Beatrice. (2004). La formación docente inicial en Chile. Recuperado de http://www.ub.edu/obipd/PDF\%20docs/Aspectes\%20laborals/Documents/La\%20Forma cion\%20Docente\%20Inicial\%20en\%20Chile.\%20AVALOS.pdf

Ávalos, Beatrice. (2009). La inserción profesional de los docentes. Profesorado: Revista de curriculum y formación del profesorado, 13(1), 43-59.

Ávalos, Beatrice, Carlson, Beverley y Aylwin, Pilar. (2004). La inserción de profesores neófitos en el sistema educativo: ¿Cuánto sienten que saben y cómo perciben su capacidad docente en relación con las tareas asignadas? (Proyecto Fondecyt N¹020218). Santiago de Chile: CONICYT.

Barber, Michael y Mourshed, Mona. (2008). Cómo hicieron los sistemas educativos con mejor desempeño del mundo para alcanzar sus objetivos. PREAL, (41), 5-48 
Bustos, Alfonso y Coll, Cesar. (2010). Los entornos virtuales como espacios de enseñanza y aprendizaje. Una perspectiva psicoeducativa para su caracterización y análisis. Revista Mexicana de Investigación Educativa, 15(44), 163-184.

Cabezas, Verónica, Gallego, Francisco, Santelices, Verónica y Zarhi, Magdalena. (2011). Factores correlacionados con las trayectorias laborales de docentes en Chile, con especial Énfasis en sus atributos académicos. Santiago: Pontificia Universidad Católica.

Castañeda, Patricia y Sánchez, María Teresa. (2009). Seguimiento de Titulados Universitarios: Análisis de perfiles de competencias inserción laboral y empleabilidad (por áreas de conocimiento) en la Universidad de Valparaíso. Revista Electrónica de Desarrollo de Competencias, 2(4), 121-131.

Centro de Estudios de Opinión Ciudadana CEOC. (2010). Encuesta de Satisfacción y Empleabilidad de Becarios Graduados MECESUP. Talca: Universidad de Talca.

Cornejo, José. (1999). Profesores que se inician en la docencia: algunas reflexiones al respecto desde América Latina. Revista Iberoamericana de Educación, (19), 51-100.

Corvalán, Oscar. (2010). La profesionalización docente, condición para la carrera docente. Revista Electrónica de Desarrollo de Competencias, 2(6), 124-151.

Cox, Cristián. (2006). Capacidades docentes para el sistema escolar: recado a las universidades. Revista UDP: pensamiento y cultura, 2(3), 58-69.

Cox, Cristián, Beca, Carlos, y Cerri, Marianela. (2011). Nueva agenda de políticas docentes en América Latina y El Caribe: Nudos críticos y criterios de acción. Santiago: OREALC /UNESCO Santiago.

Darling-Hammond, Linda y Bransford, John. (2005). Preparing teachers for a changing world: What teachers should learn and be able to do. San Francisco: Jossey-Bass Publishers.

Drinot, Michelle. (2012). La autoeficacia docente en la práctica pedagógica. (Tesis para optar el título de Licenciada en Psicología con mención en Psicología Educacional). Pontificia Universidad Católica del Perú, Lima Perú. Recuperada de http://tesis.pucp.edu.pe/repositorio/bitstream/handle/123456789/1700/DRINOT CONR OY MICHELLE AUTOEFICACIA.pdf?sequence $=1$

Enríquez, Álvaro y Rentería, Erico. (2007). Estrategias de aprendizaje para la empleabilidad en el mercado del trabajo de profesionales recién egresados. Universitas Psychologica, 6(1), 89-103.

Haydn, Terry y Barton, Roy. (2005). Common needs and different agendas: How trainee teachers make progress in their ability to use ICT subject. Some lessons from the UK. Computers y Education, 49(4), 1008-1036.

Imbernón, Francisco. (1998). La formación y el desarrollo profesional del profesorado. Hacia una nueva cultura profesional. Barcelona: Graó. 
Latorre, Marisol. (2005). Continuidades y rupturas entre Formación Inicial y Ejercicio Profesional Docente. Revista Iberoamericana de Educación, (36/2), 1-12.

Manzi, Jorge. (2010) Programa INICIA: fundamentos y primeros avances. En Cristián Bellei, Daniel Contreras y Juan Pablo Valenzuela (Eds.), Ecos de la revolución pingüina (pp.285-308). Santiago, Chile: Salesianos Impresiones, S.A.

Martínez, Francisco. (2009). El proceso de inserción laboral: implicaciones educativas para la mejora de la empleabilidad. Revista Complutense de Educación, 20(2), 455-471.

Meckes, Lorena y Bascopé, Martín. (2010). Distribución inequitativa de los nuevos profesores mejor preparados. Ponencia presentada al Primer Congreso Interdisciplinario de Investigación en Educación. Santiago, Chile: CIAE_CEPPE.

MIDE-UC. (2008). Percepción de la Calidad Actual de los Titulados y Graduados de la Educación Superior Chilena. Santiago: Pontificia Universidad Católica de Chile.

MINEDUC. (2005). Informe Comisión Sobre Formación Inicial Docente: Santiago deChile: Mineduc.

Molpeceres Pastor, Mariangeles. (2004). Identidades y formación para el trabajo en los márgenes del sistema educativo: escenarios contradictorios en la garantía social (Herramientas para la transformación, 24). Montevideo: Cinterfor/OIT.

Montecinos, Carmen, Barrios, Carolina y Tapia, María Fernanda. (2011). Relación entre estilos de supervisión durante la práctica profesional y las creencias de autoeficacia de los estudiantes de pedagogía en educación general básica. Perspectiva Educacional, 50(2), 96-122.

OCDE. (2004). Revisión de políticas nacionales de educación. París: OCDE.

Rimm-Kaufman, Sara y Sawyer, Brook. (2004). Primary-grade teachers' self-efficacy beliefs, attitudes toward teaching, and discipline and teaching practice priorities in relation to the" responsive classroom" approach. The Elementary School Journal, 104(4), 321 341.

Rojas, Francisco, Chávez, Karen y Suazo, Pablo. (2011). Transición desde el Mundo Universitario al Mundo Profesional: Redes, Necesidades y Competencias Genéricas en Estudiantes de la Universidad de La Frontera. Temuco: Centro de Innovación Profesional (CIP).

Ruffinelli, Andrea y Guerrero, Alexis. (2009). Círculo de segmentación del sistema educativo chileno: Destino laboral de egresados de Pedagogía en Educación Básica. Calidad en la Educación (31), 20-44.

Sánchez, Sergio, Fuller, Daniel y López, Miguel. (2010). Empleabilidad Temprana, Proyección de las Condiciones de Empleabilidad y Satisfacción con el Desarrollo de Competencias Genéricas en Estudiantes de la UFRO. Temuco: Centro de Innovación Profesional (CIP). 
Shulman, Lee. (1987). Knowledge and teaching: Foundations of the new reform. Harvard educational review, 57(1), 1-23.

Silva, Juan, Gros, Begoña, Garrido, José Miguel, y Rodríguez, Jaime. (2006). Estándares en tecnologías de la información y la comunicación para la formación inicial docente: situación actual y el caso chileno. Revista Iberoamericana de Educación, (38/3). Recuperado de http://www.rieoei.org/1391.htmv

Tagle, Tania, Del Valle, Rodrigo, Flores, Luzmila, y Ackley, Blaine. (2012). Las creencias de autoeficacia percibida de estudiantes de pregrado de pedagogía en inglés. Revista Iberoamericana de Educación, (58/4), 1-12.

Thieme, Claudio. (2007). El desarrollo de competencias de empleabilidad en dos universidades chilenas. Un estudio empírico. Revista OIKOS, 11(24), 47-72.

Twining, Peter, Broadie, Roger, Cook, Deirdre, Ford, Karen, Morris, David, Twiner, Alison y Underwood, Jean. (2006). Educational change and ICT: an exploration of Priorities 2 and 3 of the DfES e-strategy in schools and colleges. Conventry: Becta. Recuperado de http://oro.open.ac.uk/6408/1/Twining et al 2006 educational change and ict.pdf

Vargas, María. (2008). Perfiles de empleabilidad y desempeño profesional. Ponencia presentada al IX Congreso Nacional de Investigación Educativa. Recuperado de http://www.comie.org.mx/congreso/memoriaelectronica/v09/ponencias/at10/PRE11783 $\underline{32449 . p d f}$

Velásquez, Elda, Martínez, Loreto, y Cumsille, Patricio. (2004). Expectativas de autoeficacia y actitud prosocial asociadas a participación ciudadana en jóvenes. Psykhe (Santiago), 13(2), 85-98.

Vonk, J.H.C. (1996). A Knowledge base for mentors of beginning teachers: results of a dutch experience. In Rob McBride (Ed.), Teacher education policy (pp. 113-134). London: Falmer Press.

Walsh, Ken. (2002). IcT's about Learning: School leadership and the effective integration of information and communications technology. Recuperado de http://dera.ioe.ac.uk/5077/1/walsh-ict-full.pdf 